\title{
Diffusion-weighted MRI for imaging cell death after cytotoxic or apoptosis-inducing therapy
}

\author{
E Papaevangelou ${ }^{\star}, 1$, G S Almeida ${ }^{1}$, Y Jamin ${ }^{1}$, S P Robinson ${ }^{1}$ and N M deSouza ${ }^{1}$ \\ ${ }^{1}$ Cancer Research UK Cancer Imaging Centre, Division of Radiotherapy and Imaging, The Institute of Cancer Research and \\ The Royal Marsden NHS Foundation Trust, 15 Cotswold Road, Belmont, Sutton, Surrey SM2 5NG, UK
}

Background: Non-invasive serial imaging is desirable to detect processes such as necrotic and apoptotic cell death in cancer patients undergoing treatment. This study investigated the use of diffusion-weighted (DW-) magnetic resonance imaging (MRI) for imaging cell death induced by either a cytotoxic drug (irinotecan), or the apoptosis-inducing agent birinapant, in human tumour xenografts in vivo.

Methods: Nude mice bearing human SW620 colon carcinoma xenografts were treated with vehicle, irinotecan $\left(50 \mathrm{mg} \mathrm{kg}^{-1}\right)$ or birinapant ( $30 \mathrm{mg} \mathrm{kg}^{-1}$ ) for up to 5 days. DW-MRI was performed prior to and on days 1,3 and 5 during treatment. Assessment of tumour apoptosis and necrosis ex vivo was used to validate the imaging findings.

Results: Both irinotecan and birinapant induced significant tumour growth delay. Irinotecan induced a small increase in the tumour apparent diffusion coefficient (ADC) after 1 day, with a 20 and 30\% increase at days 3 and 5 respectively. ADC was unchanged in the vehicle- and birinapant-treated tumours despite a growth delay in the latter. Histological analysis showed that irinotecan increased necrosis at days 3 and 5, and induced apoptosis after 1 day, compared with vehicle. Birinapant induced apoptosis after day 3 , but had no effect on tumour necrosis.

Conclusions: Tumour ADC changes after irinotecan treatment were associated with the induction of a mixture of necrotic and apoptotic cell death, whereas induction of apoptosis alone with birinapant was not sufficient to induce changes in tissue microstructure that were detectable with DW-MRI. ADC is a useful non-invasive biomarker for early detection of response to cytotoxic drugs, but false negatives may arise while detecting apoptotic response to birinapant.

Effective tumour therapy necessitates the induction of cell death, which can occur through a variety of mechanisms. In particular, newer targeted agents may induce apoptosis or mitotic catastrophe rather than necrosis (Morse et al, 2007). Because of its noninvasive nature and ability to interrogate the whole tumour, imaging is crucially important in recognizing these processes while determining a therapeutic response, particularly when testing new agents in the clinic where longitudinal assessments are essential (Waterton and Pylkkanen, 2012).

Diffusion-weighted magnetic resonance imaging (DW-MRI), which measures water mobility within tissues and indirectly relates to extracellular space, has been used to provide a quantitative imaging biomarker, the apparent diffusion coefficient (ADC), of tumour response (Valonen et al, 2004; Charles-Edwards and deSouza, 2006). The low ADC, which is typical of tumours because of their high cellularity, increases in response to successful treatment as a result of tumour cell loss and extracellular expansion (Huang et al, 2008; Rozel et al, 2009). In a variety of tumour types treated with standard cytotoxic agents, for example, ovary (Kyriazi et al, 2011), and lung, esophageal, gastric, rectal cancer or liver metastasis (Sun et al, 2011), an early ADC increase after one cycle or as early as after 1 week of treatment has indicated a positive 
response to treatment. Clinical studies with targeted therapies have also shown that an ADC increase may be a potential biomarker of favourable response to treatment: a marked ADC rise at 1 week after therapy has been associated with response to the tyrosine kinase inhibitor imatinib in patients with gastrointestinal stromal tumours (Tang et al, 2011).

The mechanisms responsible for the increase in ADC following a successful therapeutic response remain poorly understood. An increase in ADC has been correlated with necrosis (Thoeny et al, 2005) and it also has been demonstrated in some studies that ADC increases as a result of morphological changes associated with apoptosis, such as cell shrinkage and membrane blebbing (Patterson et al, 2008). However, a direct comparison of the changes in ADC following cell death induced by different mechanisms has not been established. The purpose of this study, therefore, was to compare ADC changes in two different types of treatment, one a cytotoxic (irinotecan) predominantly causing necrosis and the other a novel apoptosis-inducing agent, birinapant (formerly called TL32711). SW620 tumour xenografts were imaged longitudinally, once prior to treatment, and then every other day following the treatment on up to three occasions, and imaging data validated against ex vivo analysis and histopathology.

\section{MATERIALS AND METHODS}

Cell culture. Human SW620 colorectal adenocarcinoma cells derived from lymph node metastasis were obtained from American Type Culture Collection and maintained in Dulbecco's Modified Eagle's culture medium (Sigma-Aldrich, Dorset, UK) supplemented with $2 \mathrm{~mm}$ L-glutamine, $100 \mathrm{U} \mathrm{ml}^{-1}$ penicillin, $0.2 \mathrm{mg} \mathrm{ml}^{-1}$ streptomycin and $10 \%(\mathrm{v} / \mathrm{v})$ fetal bovine serum in a humidified atmosphere with $5 \% \mathrm{CO}_{2}$ at $37^{\circ} \mathrm{C}$.

Tumour xenograft model. Animal experiments were performed in accordance with the local ethical review panel, the UK Home Office Animals (Scientific Procedures) Act 1986, and with the UK National Cancer Research Institute Guidelines for the Welfare and Use of Animals in Cancer Research (Workman et al, 2010). Female (6-8 weeks old) NCr nude mice were injected with $4 \times 10^{6}$ SW620 cells in $0.1 \mathrm{ml}$ serum-free medium subcutaneously into the flanks. Callipers were used to measure the tumour length $(L)$, width $(W)$ and depth $(D)$. Tumour volume was then calculated using the formula for an ellipsoid volume: $(\pi / 6) \times L \times W \times D$, and used to plot tumour growth curves. Approximately $2.5-3$ weeks after cell inoculation, when tumours reached a calliper volume between 200 and $250 \mathrm{~mm}^{3}$, MRI was performed (day 0 ) and tumour volume was confirmed based on MRI measurements. Mice were randomly divided into three cohorts for treatment with vehicle $(n=12)$, irinotecan $(n=11)$ or birinapant $(n=12)$.

Each mouse was treated either with $50 \mathrm{mg} \mathrm{kg}^{-1}$ irinotecan hydrochloride (Camptosar, Pfizer, Surrey, UK), $30 \mathrm{mg} \mathrm{kg}^{-1}$ birinapant (Selleck Chemicals, Houston, TX, USA) dissolved in citrate buffer, or same volume of vehicle (citrate buffer). Treatments were administered intraperitoneally on a bi-daily basis for a maximum of three doses (one dose on day 0 , one dose on day 2 and one dose on day 4). Tumours were excised on day 1 (one treatment dose), 3 (two treatment doses) or 5 (three treatment doses) ( $n=4$ per time point for each treatment, except for $n=3$ for day 5 for the irinotecan-treated cohort).

Magnetic resonance imaging. MRI measurements were performed in all mice on day 0 prior to the treatment (baseline) and on days 1 or 3 for mice killed on days 1 and 3 , respectively. In mice killed at day 5, MRI was performed at baseline and at days 1 , 3 and 5. The short-term treatment of 5 days was chosen as the main aim of this study was to find early MRI indicators of cell death in response to treatment. A 7-T horizontal bore Bruker
Micro-Imaging system (Bruker Instruments, Ettlingen, Germany) by using a $3-\mathrm{cm}$ birdcage coil was used. Anaesthesia was induced by intraperitoneal injection of a mixture of fentanyl citrate $\left(0.315 \mathrm{mg} \mathrm{ml}^{-1}\right)$ plus fluanisone $\left(10 \mathrm{mg} \mathrm{ml}^{-1}\right.$ (Hypnorm; Janssen Pharmaceutical Ltd., High Wycombe, UK)), midazolam $\left(5 \mathrm{mg} \mathrm{ml}^{-1}\right.$ (Hypnovel; Roche Applied Sciences, Burgess Hill, $\mathrm{UK})$ ) and water $(1: 1: 2)$. Mice were positioned with the tumour at the isocentre of the magnet bore and their core temperature was maintained at $37^{\circ} \mathrm{C}$ with a flow of warm air through the bore. Anatomical $\mathrm{T}_{2}$-weighted axial images were initially acquired using a rapid acquisition with refocused echoes (RARE) sequence from 20 contiguous 1 -mm-thick slices $(\mathrm{TE}=36 \mathrm{~ms}, \mathrm{TR}=4500 \mathrm{~ms}$, four averages, $128 \times 128$ phase encoding steps, $3 \times 3 \mathrm{~cm}^{2}$ field of view) for identifying the tumour centre and for quantitation of the tumour volume. A spin-echo-planar DW sequence $(\mathrm{TE}=32 \mathrm{~ms}$, $\mathrm{TR}=1500 \mathrm{~ms}$, b-values $=200-1000 \mathrm{~s} \mathrm{~mm}^{-2}, 5$ b-values, four averages) was used to determine the tumour ADC from three 1-mm-thick axial slices through the centre. After the last scan (days 1, 3 or 5 ) tumours were rapidly excised, cut axially into two parts, snap frozen on dry ice and stored at $-80^{\circ} \mathrm{C}$ to be used for ex vivo analysis.

MRI data analysis. Regions of interest (ROI) outlining the tumour were drawn on $\mathrm{T}_{2}$-weighted images in order to calculate the tumour volume at all the time points. DW-MRI data were fitted on a pixel-by pixel basis using in-house software (ImageView, developed in IDL, ITT Visual Information Systems, Boulder, CO, USA) using a Bayesian maximum a posteriori approach that took into account the Rician noise distribution to allow calculation of ADC maps (Walker-Samuel et al, 2009). The mean, median and 25th and 75th percentile ADC values were calculated from three contiguous and central tumour slices at each time point for each individual mouse.

Western blot analysis. Western blot analysis was used to measure the cleavage of the DNA repair protein poly (ADP-ribose) polymerase (PARP), which is cleaved by active caspase- 3 and indicates the middle-to-late phase of apoptosis. Tumours were homogenised in RIPA buffer (Cell Signaling, Boston, MA, USA) supplemented with protease and phosphatase inhibitors (Roche Applied Sciences). Whole and cleaved PARP were detected in the homogenates by using a rabbit anti-PARP antibody (Cell Signaling, $1: 1000)$. A rabbit anti- $\beta$-actin antibody (Cell Signaling, $1: 2000$ ) was also detected to be used as loading control. Subsequently, an anti-rabbit horseradish peroxidise-conjugated secondary antibody (Sigma-Aldrich; 1:2000) was used and signal was detected by using an enhanced chemiluminescence substrate (GE Healthcare, Buckinghamshire, UK).

Immunohistochemical analysis and in situ hybridization. Activation of caspase- 3 was used to indicate the early phase of apoptosis, whereas DNA fragmentation was used as a marker of the late/pre-necrotic phase of apoptosis.

Frozen tumour sections ( $10 \mu \mathrm{m}$ thick) were cut axially from two regions for each tumour, one in the centre of the tumour and one $1 \mathrm{~mm}$ apart, in the same orientation as the one used during MRI. Acetone fixed sections were blocked with $10 \%(\mathrm{w} / \mathrm{v})$ normal goat serum (Vector Laboratories, Peterborough, UK) in phosphatebuffered saline for $30 \mathrm{~min}$, followed by overnight incubation with a rabbit anti-cleaved caspase- 3 antibody (Abcam, Cambridge, UK, $1: 20)$. A non-immune-specific rabbit IgG was used in the same concentration with the anti-cleaved caspase- 3 antibody, as a negative isotype control. The following day, slides were washed twice and incubated for $1 \mathrm{~h}$ with an Alexa-Fluor 546 goat antirabbit secondary antibody (Invitrogen, Paisley, UK, 1:1000). Fluorescent staining was visualised at $556-573 \mathrm{~nm}$ on a BX51 microscope (Olympus Optical, London, UK) and a motorised scanning stage (Prior Scientific Instruments, Cambridge, UK) 
driven by image analysis software CellP (Soft Imaging System, Munster, Germany) to record digital composite images of whole tumour sections.

DNA double strand breaks were detected with an in situ cell death detection kit (Roche Applied Sciences) using a Terminal deoxynucleotidyl Transferase (TdT)-mediated deoxyuridine triphosphate (dUTP) nick end labelling (TUNEL) approach. Paraformaldehyde fixed and permeabilized sections were incubated with a mixture of enzyme (TdT) and fluorescein labelled nucleotide (dUTP) for $1 \mathrm{~h}$. Sections were also incubated with label solution without the terminal transferase, as a negative control. Fluorescent staining was detected at $515-565 \mathrm{~nm}$ and composite whole tumour sections were recorded.

Sections were also stained with haematoxylin and eosin (H\&E), dehydrated through a series of alcohols and xylene and visualized under light. Composite images of whole tumour sections were recorded.

The image analysis software ImageJ (Schneider et al., 2012) was used for post-processing of all digital composite tumour images. Post-processing was blinded to the type and duration of treatment. For cleaved caspase-3 and TUNEL staining, ROIs encompassing the whole tumour sections were defined and fluorescent particles detected above a constant intensity threshold across all tumour sections, and the area of tumour with fluorescent staining expressed as a percentage of the whole tumour section area. The intensity threshold was set above background staining to associate with positive staining only; the background fluorescent intensity staining was defined initially by using negative control stained sections. Digital composite images from the H\&E sections were used to quantify the degree of tumour necrosis. Using ImageJ, all necrotic areas or foci in each tumour section were measured by drawing ROIs and expressed as a percentage of the whole tumour section area.

Statistical analysis. GraphPad Prism 5 (GraphPad Software Inc., La Jolla, CA, USA) was used to perform statistical analysis. The mean values for tumour volume, mean of median values for ADC, the mean necrotic and stained area fractions, and the mean integrated density of protein bands were used for all the mice in each time point. The variability in baseline tumour ADC across the cohort was documented. To validate the reproducibility of the technique, the coefficient of variation $(\mathrm{CoV})$ between two baseline measurements made in seven mice scanned $24 \mathrm{~h}$ apart was calculated. Any significant differences in measured parameters between vehicle- and drug-treated tumours were identified by using one-way ANOVA with Bonferroni post test, with a $5 \%$ level of significance. Frequency distribution analysis was also used for all ADC values of each tumour. Results are presented as the mean \pm 1 s.e.m.

\section{RESULTS}

Treatment with the cytotoxic drug irinotecan or the apoptosisinducing agent birinapant caused a significant tumour growth delay in SW620 colon cancer xenografts. SW620 tumours had a doubling rate of $3.3 \pm 0.6$ days based on independent tumour volume growth curves for each of the mice used in this study, where the volume of the tumours was calculated from calliper dimension measurements. Calliper volume and MRI volume measurements were very similar and no statistical significance was found when a paired $t$-test was performed in over 92 tumour volumes calculated with both methods. The mean MRI tumour volume at day 0 was $201 \pm 47 \mathrm{~mm}^{3}$.

Both irinotecan and birinapant treatment induced a significant growth delay as assessed by MRI-derived tumour volume measurements in comparison with controls from day 3 onwards.
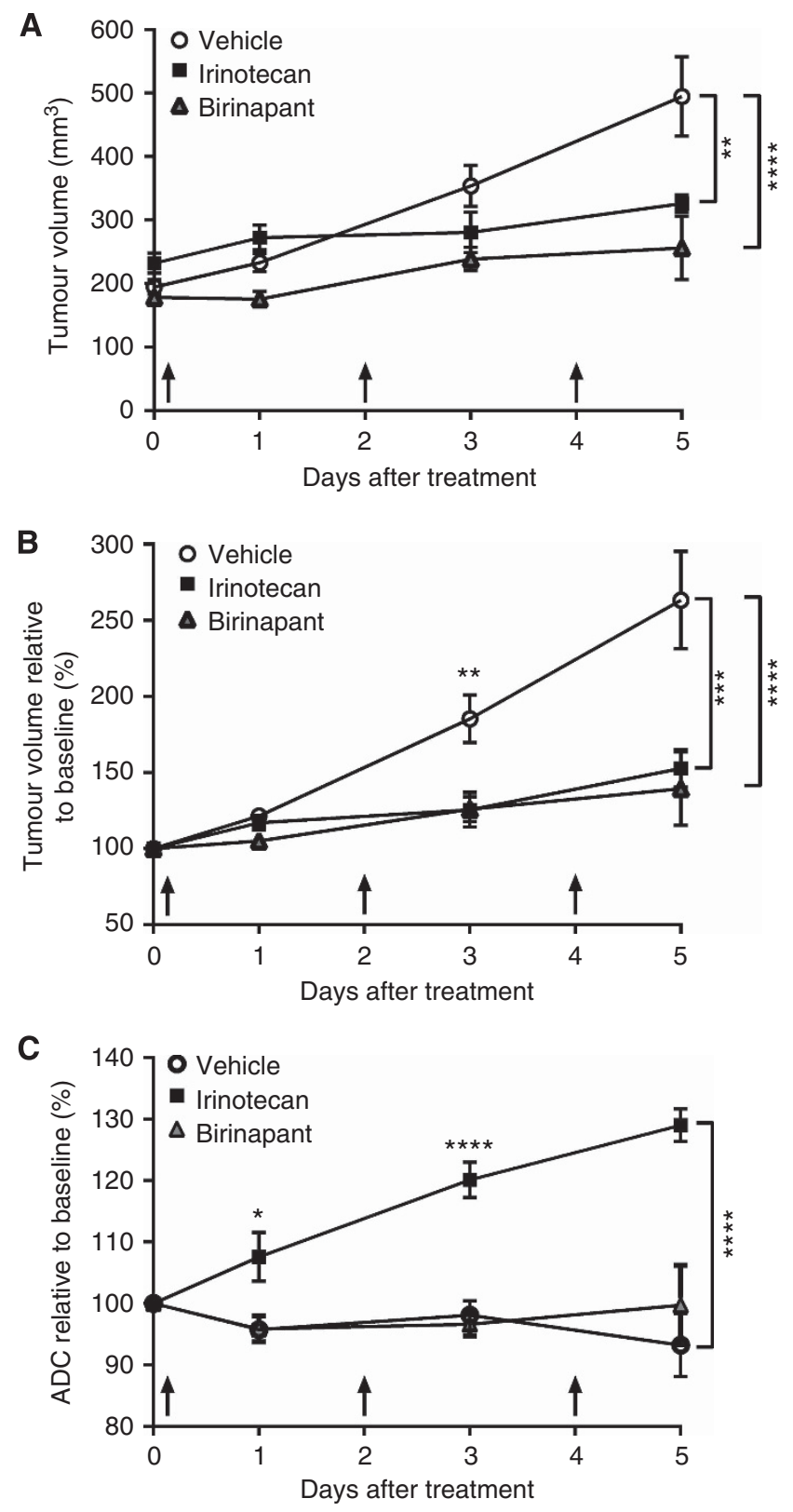

Figure 1. The anti-tumour effects of birinapant or irinotecan therapy in SW620 human colon adenocarcinoma xenografts. (A) Actual tumour volume changes and (B) tumour volume changes relative to baseline (day 0 ) over the course of treatment show an increase in tumour volume in the vehicle-treated cohort, while both drugs (birinapant and irinotecan) induced significant tumour growth delay. (C) Changes in tumour ADC relative to baseline in vehicle- and drug-treated cohorts over 5 days. Black arrows indicate the dosing schedule. Data are mean for tumour volumes or mean of median values for $A D C \pm 1$ s.e.m., ${ }^{\star} P<0.05,{ }^{\star *} P<0.01,{ }^{\star \star *} P<0.001,{ }^{\star} * \star \star * P<0.0001$.

As shown in Figure 1B, the volume of the vehicle-treated tumours increased by $85 \pm 16 \%$ at day 3 , and by $163 \pm 32 \%$ at day 5 , while the volume of the irinotecan-treated cohort increased only by $26 \pm 11 \%$ at day 3 , and by $52 \pm 12 \%$ at day 5 . The birinapanttreated tumours increased in volume by $26 \pm 8 \%$ at day 3 , and by $40 \pm 24 \%$ at day 5 .

ADC increases in response to cytotoxic treatment at day 1 prior to tumour growth delay, whereas apoptotic-specific treatment had no effect on ADC. Means of the median ADC values are given in Figure 1C. The baseline variability in ADC across all 

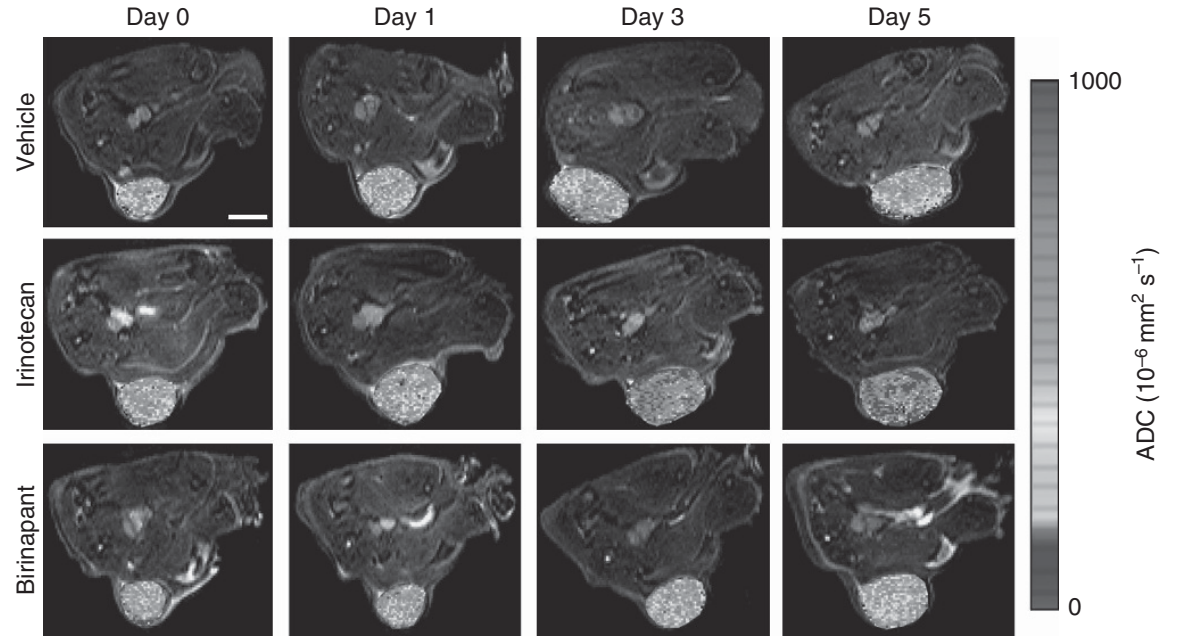

Figure 2. Diffusion MRI is sensitive to early irinotecan treatment in SW620 xenografts. Representative $\mathrm{T}_{2}$-weighted anatomic MRI images overlaid with parametric ADC maps acquired prior to (day 0) and at 1, 3 and 5 days following treatment with vehicle, irinotecan or birinapant in SW620 tumours. Scale bar $5 \mathrm{~mm}$. A full color version of this figure is available at British Journal of Cancer journal online.

tumours was 6\%. The $\mathrm{CoV}$ of repeated ADC measurements in seven mice scanned on two consecutive days was $4 \%$.

Representative ADC maps overlaid on DW images of a vehicle-, an irinotecan- and a birinapant-treated tumour at baseline and at days 1, 3 and 5 post treatment are shown in Figure 2; there was an increase in ADC in the irinotecan-treated tumour at days 3 and 5. Figure 3 shows the mean ADC frequency distribution scatter plots of all tumours in each of the three cohorts, indicating that irinotecan causes a shift of the ADC distribution towards higher values at days 3 and 5. Tumour ADC was significantly higher at days 1,3 and 5 post treatment with irinotecan by $8 \%(P<0.05)$, $20 \%(P<0.0001)$ and $30 \%(P<0.0001)$, respectively; the vehicleand birinapant-treated cohorts only changed by $\pm 5 \%$ over the course of the treatment. Table 1 summarises the ADC data for days $0,1,3$ and 5 . The median ADC value of the irinotecan-treated cohort increased to 665 and $700 \times 10^{-6} \mathrm{~mm}^{2} \mathrm{~s}^{-1}$ at days 3 and 5 respectively, whereas in the vehicle-treated cohort the median ADC value was 533 and $513 \times 10^{-6} \mathrm{~mm}^{2} \mathrm{~s}^{-1}$ at days 3 and 5 , respectively. In the birinapant-treated cohort the median ADC value was 509 and $531 \times 10^{-6} \mathrm{~mm}^{2} \mathrm{~s}^{-1}$ at days 3 and 5 respectively.

The irinotecan-induced change in ADC in the SW620 tumours is associated with necrotic and apoptotic cell death. Figure 4 shows representative histological sections of excised tumours after 5 days of treatment with vehicle, irinotecan or birinapant, stained with H\&E (Figure 4A), TUNEL staining (Figure 4B) and cleaved caspase-3 staining (Figure 4C). Quantification of tumour necrosis using the H\&E stained sections at days 1,3 or 5 after treatment is shown in Figure 5A. The irinotecan-treated tumours displayed a significantly higher degree of necrosis $(32 \pm 6 \%$ and $31 \pm 8 \%$, $P<0.05)$, compared with vehicle-treated tumours $(17 \pm 4$ and $18 \pm 3 \%$ ), on both days 3 and 5 . Necrosis was also higher, $23 \pm 2 \%$, in the birinapant-treated cohort at day 5 compared with the vehicle-treated tumours, but the results were not statistically significant.

At day 3, TUNEL staining was significantly greater in both the irinotecan $(14 \%, P<0.001)$ and birinapant $(11 \%, P<0.01)$ treated cohorts compared with the vehicle-treated group (5\%) (Figure $5 \mathrm{C}$ ). At day 5, TUNEL staining was $11 \pm 1 \%(P<0.01)$ in the irinotecan-treated and $10 \pm 2 \%(P<0.05)$ in the birinapant-treated cohorts compared with $7 \pm 1 \%$ in the vehicle-treated group.

Caspases were activated after treatment with either irinotecan or birinapant (Figure 5B). Cleaved caspase-3 positive staining was
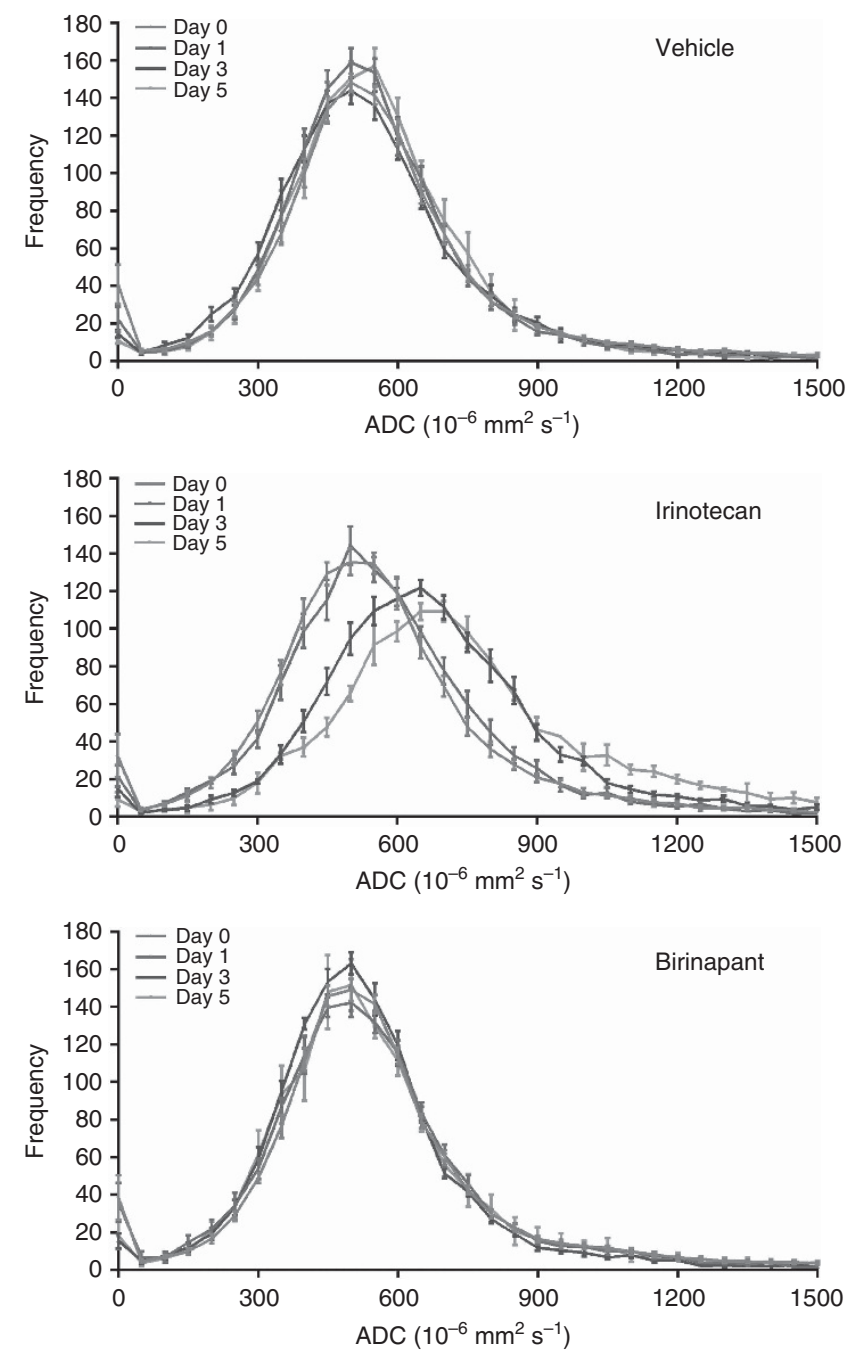

Figure 3. The effects of irinotecan or birinapant treatment on ADC in SW620 xenografts. Frequency scatter plots show the distribution of the tumour ADC values prior to and at days 1, 3 and 5 after treatment with vehicle, irinotecan or birinapant. ADC frequency values were calculated across three MRI slices for each mouse and data are the mean \pm 1 s.e.m. frequency values of all mice in each cohort at each time point. 
Table 1. Summary of the mean, 25th percentile $\left(Q_{1}\right)$, median and 75th percentile $\left(Q_{3}\right)$ ADC values in SW620 tumours at day 0 (prior to treatment), and days 1, 3 and 5 after treatment with vehicle, irinotecan or birinapant

\begin{tabular}{|c|c|c|c|c|c|}
\hline & & & ADC & $\left.s^{-1}\right)$ & \\
\hline & Treatment & Mean & $\mathrm{Q}_{1}$ & Median & $\mathrm{Q}_{3}$ \\
\hline Day 0 & Vehicle $(n=12)$ & $946 \pm 138$ & $430 \pm 10$ & $538 \pm 10$ & $671 \pm 15$ \\
\hline & Irinotecan $(n=11)$ & $755 \pm 72$ & $422 \pm 12$ & $535 \pm 13$ & $678 \pm 23$ \\
\hline & Birinapant $(n=12)$ & $849 \pm 60$ & $426 \pm 5$ & $531 \pm 7$ & $665 \pm 15$ \\
\hline Day 1 & Vehicle $(n=8)$ & $835 \pm 117$ & $417 \pm 7$ & $515 \pm 8$ & $628 \pm 11$ \\
\hline & Irinotecan $(n=7)$ & $883 \pm 84$ & $437 \pm 14$ & $555 \pm 15$ & $609 \pm 31$ \\
\hline & Birinapant $(n=8)$ & $970 \pm 148$ & $397 \pm 16$ & $513 \pm 12$ & $661 \pm 11$ \\
\hline Day 3 & Vehicle $(n=8)$ & $839 \pm 106$ & $420 \pm 10$ & $533 \pm 12$ & $670 \pm 18$ \\
\hline & Irinotecan $(n=7)$ & $987 \pm 142$ & $535 \pm 9 * \star \star \star$ & $665 \pm 11^{\star * * *}$ & $814 \pm 16^{\star \star \star \star}$ \\
\hline & Birinapant $(n=8)$ & $737 \pm 53$ & $410 \pm 8$ & $509 \pm 6$ & $612 \pm 14^{\star}$ \\
\hline Day 5 & Vehicle $(n=4)$ & $704 \pm 100$ & $406 \pm 13$ & $513 \pm 9$ & $627 \pm 12$ \\
\hline & Irinotecan $(n=3)$ & $1071 \pm 191$ & $562 \pm 20^{\star \star \star}$ & $700 \pm 17^{\star \star \star}$ & $880 \pm 30$ ** \\
\hline & Birinapant $(n=4)$ & $845 \pm 106$ & $423 \pm 20$ & $531 \pm 29$ & $671 \pm 52$ \\
\hline
\end{tabular}

A

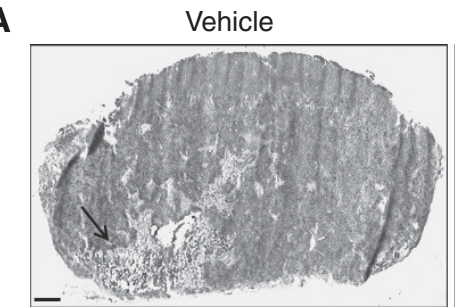

B

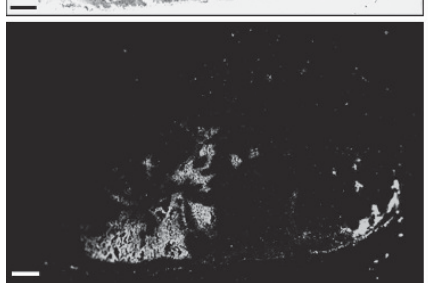

C

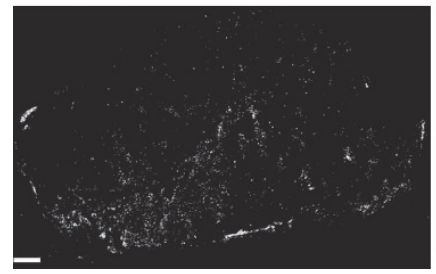

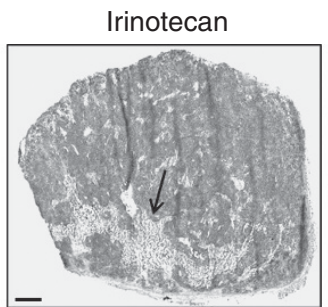
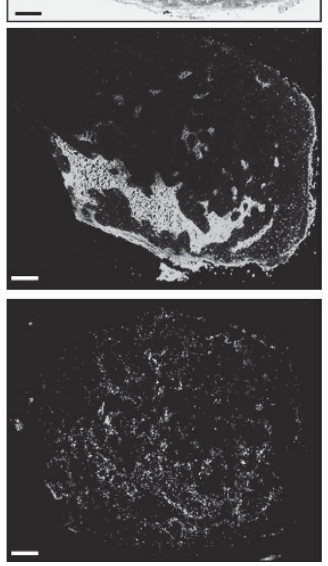

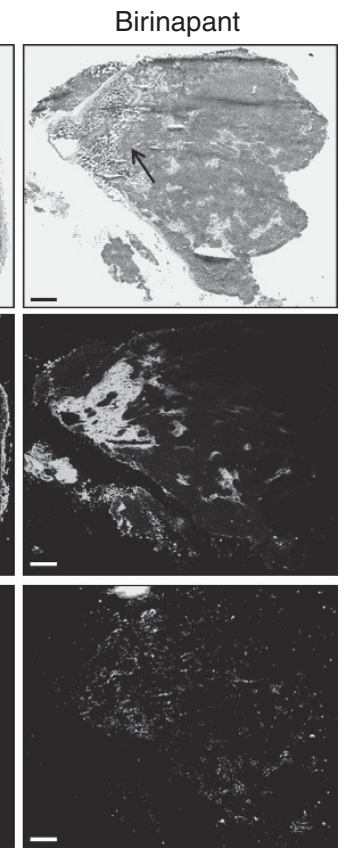

Figure 4. Histological assessment of cell death in SW620 xenografts at day 5 after treatment with vehicle, irinotecan or birinapant. (A) Composite images of H\&E-stained sections indicating necrotic regions (arrows). (B) Composite images from frozen tumour sections stained with the fluorescein in situ cell death detection kit. Areas with DNA fragmentation fluoresce green. (C) Composite images from frozen sections stained with the apoptotic marker cleaved caspase-3, detected using an Alexa-546-conjugated secondary antibody that fluoresces red. Scale bar $0.5 \mathrm{~mm}$. A full color version of this figure is available at British Journal of Cancer journal online.

increased in the irinotecan-treated cohort to $2 \%$ in all days ( 1,3 and 5 ) compared to $<0.8 \%$ in the vehicle-treated group. In the birinapant-treated tumours there was an increase in positive staining to more than $2 \%$ at day 3 compared with $0.8 \pm 0.2 \%$ in the vehicle $(P<0.001)$, but it decreased to $1.2 \pm 0.3 \%$ at day 5 despite still being higher than in the vehicle-treated tumours $(P<0.05)$.

Western blot analysis performed on tumour extracts for PARP and cleaved PARP protein expression (Figure 5D) shows reduction of whole PARP and increase in cleaved PARP protein expression in both treated cohorts at day 5, confirming the downstream signalling as a result of apoptosis; however, the effect was more profound in the irinotecan-treated tumours. Densitometry analysis of the protein bands for cleaved PARP for all time points (Figure 5E) shows an increase in PARP cleavage on day 3 for tumours treated with irinotecan or birinapant $(P<0.05$ and
$P<0.01$, respectively) compared with vehicle-treated tumours. On day 5, PARP cleavage was even higher on the irinotecantreated cohort $(P<0.001)$ compared with the vehicle-treated. Cleaved PARP was also increased on day 5 on the birinapanttreated tumours but the result was not significantly different $(P>0.05)$ from the vehicle-treated cohort. The ex vivo findings suggest that irinotecan results in a mixture of necrotic and apoptotic cell death, whereas birinapant causes cell death via the apoptotic pathway.

\section{DISCUSSION}

In this study, we have exploited the use of DW-MRI for evaluating response to two anticancer agents, birinapant and irinotecan, 

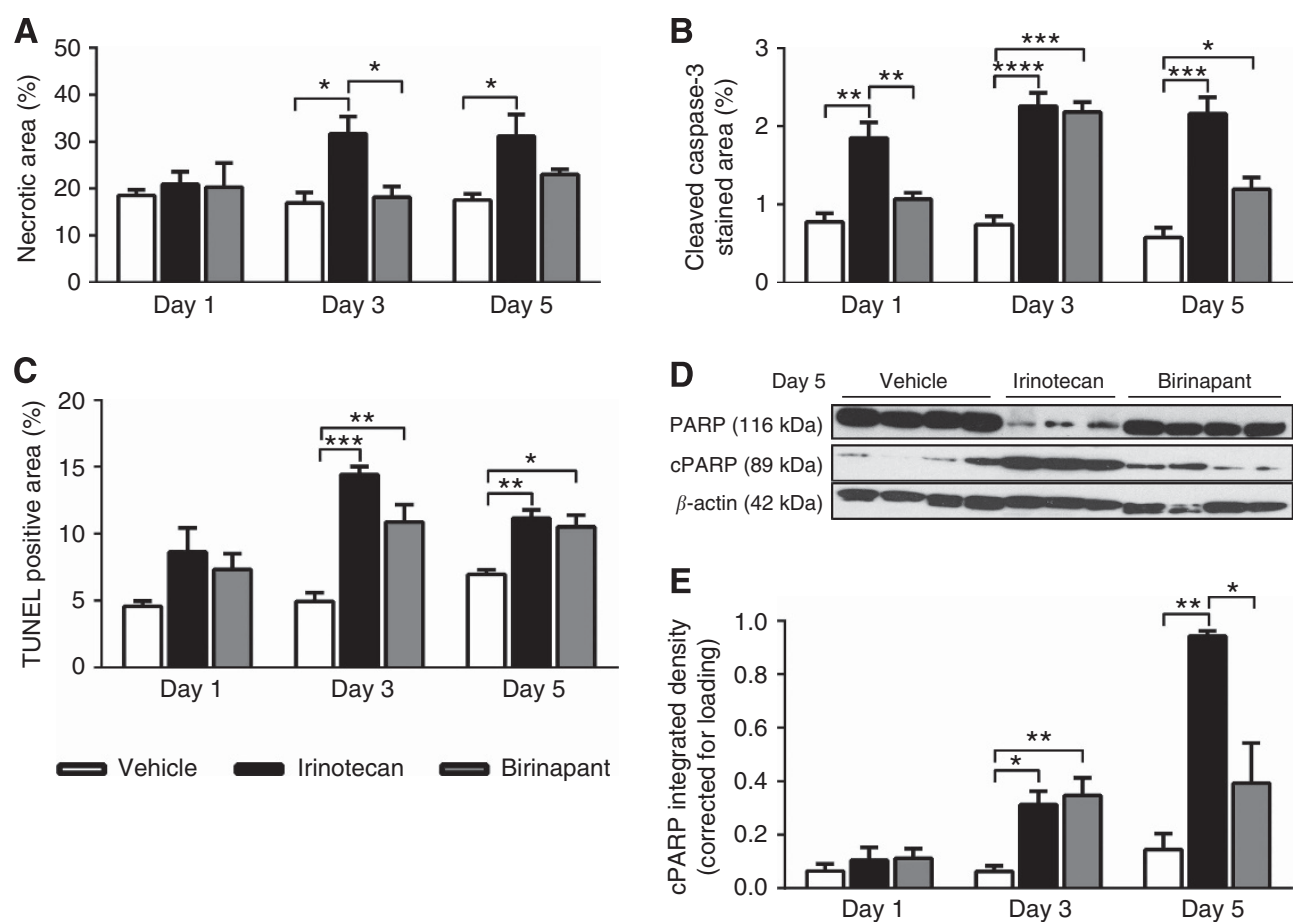

Figure 5. Ex vivo assessment and quantification of histological markers of cell death in SW620 xenografts treated with vehicle, irinotecan or birinapant. (A) Necrotic area, (B) cleaved caspase-3 positive area, and (C) TUNEL positive area. Results are means +1 s.e.m of two sections per tumour for $n=4$ per time point of each treatment, except for $n=3$ at day 5 in the irinotecan-treated cohort. (D) Western blot from tumours harvested at day 5 after treatment showing an increase in PARP cleavage in the irinotecan-treated group. (E) Densitometry of western blots corrected by using $\beta$-actin for tumours harvested at days 1, 3 and 5 after treatment showing an increase in PARP cleavage in irinotecan- or birinapant-treated compared to vehicle-treated tumours after day $3 .{ }^{\star} P<0.05,{ }^{\star \star} P<0.01,{ }^{\star \star \star} P<0.001,{ }^{\star \star \star \star \star} P<0.0001$.

which were predicted to elicit two different types of response, apoptosis for birinapant and a mixture of necrosis and apoptosis for irinotecan. The dosages of both agents were based on previous publications. Irinotecan at a dose of $50 \mathrm{mg} \mathrm{kg}^{-1}$ has been shown to induce tumour growth delay on SW620 xenografts (Zabludoff et al, 2008), and birinapant at a dose of $30 \mathrm{mg} \mathrm{kg}^{-1}$ had a similar effect on human melanoma xenografts and in patient-derived xenotransplant models of ovarian cancer, colorectal cancer and melanoma (Krepler et al, 2013; Benetatos et al, 2014).

DW-MRI used to monitor changes in tumour water diffusion as a result of cell death caused by two treatments with different mechanisms of action showed a striking increase in ADC in the presence of necrosis, but no significant increase above baseline variation when purely apoptosis was present. Although the increase in ADC after 3 and 5 days of irinotecan treatment compared with the vehicle was more profound, a small increase in tumour ADC could be observed following this treatment even after one day. This suggests that the tissue changes that precede the frank histological recognition of necrosis (as confirmed by ex vivo analysis) cause an increase in water diffusivity. However, irinotecan treatment also led to increased apoptosis as revealed with TUNEL and cleaved caspase- 3 staining, and cleaved PARP western blot analysis. Hence, irinotecan caused an ADC increase as a result of a mixture of necrotic and apoptotic cell death. This is in agreement with a previous study indicating that the increase in tumour ADC is associated with induction of global cell death as a result of various mechanisms, rather than only a specific form of cell death (Morse et al, 2007). In a mouse lymphoma model, irinotecan was also shown to increase ADC by $14 \%$ after 2 days of treatment; this change in ADC correlated with increased apoptosis and decreased tumour proliferation ( $\mathrm{Ng}$ et al, 2013).

In contrast, tumour growth delay caused by birinapant from day 3 (due to induction of apoptosis as confirmed by ex vivo analysis), although equivalent to that induced by irinotecan, was not accompanied by an increase in ADC. The increase in tumour cleaved caspase- 3 staining and cleaved PARP protein expression in the birinapant-treated cohort compared with the vehicle-treated, and DNA fragmentation (as indicated by TUNEL staining, which also increased on days 3 and 5 after birinapant treatment), confirmed birinapant as a clear apoptotic-inducing agent. Importantly, necrosis did not change significantly. Therefore, although birinapant-treated tumours grew more slowly and underwent apoptosis, the lack of change in water diffusivity suggests that at these early time-points tissue microstructure has not been compromised. This is of crucial importance when considering newer targeted agents which often predominantly cause apoptosis. In these situations, the ADC is unlikely to increase early unless extensive necrosis is also present or significant disruption to tissue microstructure occurs. Other studies have confirmed the need for extensive regions of necrosis before an increase in tumour ADC can be expected (Lyng et al, 2000; Bohndiek et al, 2010). It may well be that changes because of an extensive necrosis cause a more substantial disruption in tissue microarchitecture and hence more dramatic and acute alteration in water diffusion, whereas the effect of apoptosis is smaller, and more diffuse and homogeneously distributed in the tumour area, and thus might be undetectable in ADC measurements made at early time points. One possible explanation why treatment with birinapant has no effect on water diffusivity or on tissue microarchitecture might be that removal of the dead cells is followed by tumour cell repopulation. A study has shown that the apoptotic process can stimulate the repopulation of tumours undergoing radiotherapy and that this process is mediated by activated caspase-3 (Huang et al, 2011). In our study, tumour cell repopulation could have occurred after both treatments due to increased caspase activation, as the levels of cleaved caspase- 3 were comparable 
between treatments. However, the repopulation effect would be more evident in the case of birinapant only, because in the case of irinotecan the increased necrosis (almost double compared with birinapant) would mask such an effect. Hence, when monitoring cytotoxic therapy, DW-MRI can be used early on during treatment to predict treatment response but when imaging targeted therapies, DW-MRI may be poorly sensitive for measuring early response; the timing of imaging is crucial to eliminate the risk of false-negative results (Sinkus et al, 2012).

Preclinical studies of targeted agents have reported ADC increase following treatment with the HIF inhibitor NSC-134754 at $24 \mathrm{~h}$ (Baker et al, 2012), the Jak1/2 inhibitor AZD1480 at 3 days (Loveless et al, 2012), and the MEK1/2 inhibitor selumetinib at 3 days (Beloueche-Babari et al, 2013). The changes in water diffusion in these studies were associated with an increase in the extracellular space due to the induction of necrosis or a mixture of cell death, which is in agreement with our study in the case of irinotecan. In mouse breast cancer xenografts, treatment with the death receptor 4 antibody TRA-8, an apoptosis-inducing drug, led to an increase in ADC after 3 days of treatment, which was linearly proportional to the apoptotic cell and caspase- 3 densities and inversely proportional to the proliferating cell density; however, ADC started to decrease after 6 days of treatment (Kim et al, 2008). In our study no changes in ADC were observed after treatment with the apoptosis-inducing birinapant during the 5 days of treatment duration; however, in the study by Kim et al, induction of caspase-3 activation was 10-fold higher than in our study.

The lag time between exposure to the drug and the appearance of morphological changes of cell death is likely to be highly variable and depends heavily on the cell and tumour type, the type of treatment, its duration and intensity and the local environmental conditions of the cells (Martin et al, 1995), hence a time course monitoring of the treatment response was chosen for a duration of 5 days, which included a maximum of four MRI sessions per tumour. Tumours excised from mice treated with the same drug but on different time points could be undergoing a different stage of apoptosis. This could explain the slightly higher degree of DNA fragmentation seen by TUNEL staining in day 3 compared with day 5 . The study duration was confined to 6 days (including baseline), as the SW620 tumour doubling times were $3.3 \pm 0.6$ days, which meant that the vehicle-treated animals reached the Home Office tumour size restriction limits over this time. Furthermore, necrosis in the vehicle-treated group of $18 \%$ at day 5 (Figure 5A) was observed in this SW620 subcutaneous tumour model, which was maintained at the same level for the duration of the treatment.

A variety of imaging modalities have been used to image treatment response. Increased activation of caspases during apoptosis can be visualised by using the PET radiolabelled selective inhibitor of activated caspase-3 and -7, isatin 5-sulphonamide ([18F]ICMT-11) (Nguyen et al, 2009), and fluorescent or radiolabelled annexin $\mathrm{V}$, a natural PS-binding protein, is widely used to monitor PS exposure on the cell membrane (Collingridge et al, 2003; Glaser et al, 2003). High-frequency ultrasound has been shown to detect nuclear fragmentation and DNA clumping (Vlad et al, 2010), while accumulation of lipid droplets has been identified with proton magnetic resonance spectroscopy (Delikatny et al, 2011; Zietkowski et al, 2012). A recent study has also shown the potential of magnetic resonance elastography for imaging treatment-induced tumour necrosis ( $\mathrm{Li}$ et al, 2014). However, of the available techniques, DW-MRI is routinely used in the clinic and is easy to implement. Its role in drug development is therefore escalating.

We selected a potent cytotoxic and a pure apoptotic agent for our comparative study. Irinotecan, a topoisomerase inhibitor, prevents DNA unwinding and results in cell death due to DNA damage (Guichard et al, 1999). As a single agent or in combination with other drugs, it has been previously shown to cause tumour growth delay in SW620 tumours (Davies et al, 2007; Zabludoff et al, 2008), and also to induce tumour necrosis factor production leading to apoptotic cell death via the extrinsic pathway (Goto et al, 1996). Moreover, it can induce caspase-3-like protease activity in vitro in SW620 cells (Bergmann-Leitner and Abrams, 2001). On the other hand, birinapant, a mimetic of the second mitochondriaderived activator of caspases, has shown in vivo antitumor activity in melanomas, MDA-MB-231 breast cancer tumours and primary patient-derived xenotransplant models of human ovarian cancer, melanoma and colorectal cancer (Krepler et al, 2013; Benetatos et al, 2014; Condon et al, 2014), by antagonising the inhibitors of apoptosis proteins and activating caspases (Allensworth et al, 2013). Birinapant treatment for $6 \mathrm{~h}$ of HCT116 colon carcinoma, or MDA-MB-231 breast adenocarcinoma tumours resulted in a temporal increase in caspase- 3 activation evidenced by increased uptake of $\left[{ }^{18} \mathrm{~F}\right] \mathrm{ICMT}-11$, which was subsequently reduced at $24 \mathrm{~h}$ post treatment (Nguyen et al, 2013). Clinical benefit has been demonstrated when birinapant was combined with irinotecan in patients with irinotecan-refractory colorectal cancer (Senzer et al, 2014). We therefore selected these agents singly and studied their effects in vivo in a colorectal tumour model.

In conclusion, we have demonstrated that irinotecan causes an increase in tumour ADC in a colon carcinoma xenograft, which was associated primarily with induction of necrosis with associated apoptotic cell death, and could be measured as early as $24 \mathrm{~h}$ post treatment prior to changes in the tumour volume. Induction of apoptosis in the same tumour model as a result of treatment with birinapant was not sufficient to induce measurable changes in ADC within 5 days of the treatment, despite the drug suppressing tumour growth. Thus, tumour ADC appears to be a promising, clinically translatable, imaging biomarker that can be used for early and non-invasive detection of therapeutic response to cytotoxic drugs. However, when considering apoptotic cell death in response to targeted therapeutics, use of ADC could potentially result in false negatives, particularly if imaging at an early time point before significant disruption to tissue microarchitecture has occurred.

\section{ACKNOWLEDGEMENTS}

We thank Dr J. Bamber, Dr S. Eccles, Dr G. Smith and Dr Y-L. Chung at the ICR for critical discussions, and the staff at the Biological Services Unit, ICR, for animal care and maintenance. This work was supported by the Cancer Research UK and the EPSRC Cancer Imaging Centre in association with the MRC and Department of Health (England) (Grants C1060/A10334).

\section{CONFLICT OF INTEREST}

The authors declare no conflict of interest.

\section{REFERENCES}

Allensworth JL, Sauer SJ, Lyerly HK, Morse MA, Devi GR (2013) Smac mimetic Birinapant induces apoptosis and enhances TRAIL potency in inflammatory breast cancer cells in an IAP-dependent and TNF-alphaindependent mechanism. Breast Cancer Res Treat 137: 359-371.

Baker LC, Boult JK, Walker-Samuel S, Chung YL, Jamin Y, Ashcroft M, Robinson SP (2012) The HIF-pathway inhibitor NSC-134754 induces metabolic changes and anti-tumour activity while maintaining vascular function. Br J Cancer 106: 1638-1647. 
Beloueche-Babari M, Jamin Y, Arunan V, Walker-Samuel S, Revill M, Smith PD, Halliday J, Waterton JC, Barjat H, Workman P, Leach MO, Robinson SP (2013) Acute tumour response to the MEK1/2 inhibitor selumetinib (AZD6244, ARRY-142886) evaluated by non-invasive diffusion-weighted MRI. Br J Cancer 109: 1562-1569.

Benetatos CA, Mitsuuchi Y, Burns JM, Neiman EM, Condon SM, Yu G, Seipel ME, Kapoor GS, Laporte MG, Rippin SR, Deng Y, Hendi MS, Tirunahari PK, Lee YH, Haimowitz T, Alexander MD, Graham MA, Weng D, Shi Y, McKinlay MA, Chunduru SK (2014) Birinapant (TL32711), a bivalent SMAC mimetic, targets TRAF2-associated cIAPs, abrogates TNF-induced NF-kappaB activation, and is active in patient-derived xenograft models. Mol Cancer Ther 13: 867-879.

Bergmann-Leitner ES, Abrams SI (2001) Treatment of human colon carcinoma cell lines with anti-neoplastic agents enhances their lytic sensitivity to antigen-specific CD8 + cytotoxic T lymphocytes. Cancer Immunol Immunother 50: 445-455.

Bohndiek SE, Kettunen MI, Hu DE, Witney TH, Kennedy BW, Gallagher FA, Brindle KM (2010) Detection of tumor response to a vascular disrupting agent by hyperpolarized 13C magnetic resonance spectroscopy. Mol Cancer Ther 9: 3278-3288.

Charles-Edwards EM, deSouza NM (2006) Diffusion-weighted magnetic resonance imaging and its application to cancer. Cancer Imaging 6: $135-143$.

Collingridge DR, Glaser M, Osman S, Barthel H, Hutchinson OC, Luthra SK, Brady F, Bouchier-Hayes L, Martin SJ, Workman P, Price P, Aboagye EO (2003) In vitro selectivity, in vivo biodistribution and tumour uptake of annexin $\mathrm{V}$ radiolabelled with a positron emitting radioisotope. $\mathrm{Br} J$ Cancer 89: $1327-1333$.

Condon SM, Mitsuuchi Y, Deng Y, LaPorte MG, Rippin SR, Haimowitz T, Alexander MD, Kumar PT, Hendi MS, Lee YH, Benetatos CA, Yu G, Kapoor GS, Neiman E, Seipel ME, Burns JM, Graham MA, McKinlay MA, Li X, Wang J, Shi Y, Feltham R, Bettjeman B, Cumming MH, Vince JE, Khan N, Silke J, Day CL, Chunduru SK (2014) Birinapant, a smac-mimetic with improved tolerability for the treatment of solid tumors and hematological malignancies. J Med Chem 57: 3666-3677.

Davies BR, Logie A, McKay JS, Martin P, Steele S, Jenkins R, Cockerill M, Cartlidge S, Smith PD (2007) AZD6244 (ARRY-142886), a potent inhibitor of mitogen-activated protein kinase/extracellular signal-regulated kinase kinase 1/2 kinases: mechanism of action in vivo, pharmacokinetic/ pharmacodynamic relationship, and potential for combination in preclinical models. Mol Cancer Ther 6: 2209-2219.

Delikatny EJ, Chawla S, Leung DJ, Poptani H (2011) MR-visible lipids and the tumor microenvironment. NMR Biomed 24: 592-611.

Glaser M, Collingridge DR, Aboagye EO, Bouchier-Hayes L, Hutchinson OC, Martin SJ, Price P, Brady F, Luthra SK (2003) Iodine-124 labelled annexin- $\mathrm{V}$ as a potential radiotracer to study apoptosis using positron emission tomography. Appl Radiat Isot 58: 55-62.

Goto S, Okutomi T, Suma Y, Kera J, Soma G, Takeuchi S (1996) Induction of tumor necrosis factor by a camptothecin derivative, irinotecan, in mice and human mononuclear cells. Anticancer Res $\mathbf{1 6}$ 2507-2511.

Guichard S, Terret C, Hennebelle I, Lochon I, Chevreau P, Frétigny E, Selves J, Chatelut E, Bugat R, Canal P (1999) CPT-11 converting carboxylesterase and topoisomerase activities in tumour and normal colon and liver tissues. Br J Cancer 80: 364-370.

Huang MQ, Pickup S, Nelson DS, Qiao H, Xu HN, Li LZ, Zhou R, Delikatny EJ, Poptani H, Glickson JD (2008) Monitoring response to chemotherapy of non-Hodgkin's lymphoma xenografts by T(2)-weighted and diffusion-weighted MRI. NMR Biomed 21: 1021-1029.

Huang Q, Li F, Liu X, Li W, Shi W, Liu FF, O’Sullivan B, He Z, Peng Y, Tan AC, Zhou L, Shen J, Han G, Wang XJ, Thorburn J, Thorburn A, Jimeno A, Raben D, Bedford JS, Li CY (2011) Caspase 3-mediated stimulation of tumor cell repopulation during cancer radiotherapy. Nat Med 17: 860-866.

Kim H, Morgan DE, Zeng H, Grizzle WE, Warram JM, Stockard CR, Wang D, Zinn KR (2008) Breast tumor xenografts: diffusion-weighted MR imaging to assess early therapy with novel apoptosis-inducing anti-DR5 antibody. Radiology 248: 844-851.

Krepler C, Chunduru SK, Halloran MB, He X, Xiao M, Vultur A, Villanueva J, Mitsuuchi Y, Neiman EM, Benetatos C, Nathanson KL, Amaravadi RK, Pehamberger H, McKinlay M, Herlyn M (2013) The novel SMAC mimetic birinapant exhibits potent activity against human melanoma cells. Clin Cancer Res 19: 1784-1794.

Kyriazi S, Collins DJ, Messiou C, Pennert K, Davidson RL, Giles SL, Kaye SB, Desouza NM (2011) Metastatic ovarian and primary peritoneal cancer: assessing chemotherapy response with diffusion-weighted MR imaging-value of histogram analysis of apparent diffusion coefficients. Radiology 261: 182-192.

Li J, Jamin Y, Boult JK, Cummings C, Waterton JC, Ulloa J, Sinkus R, Bamber JC, Robinson SP (2014) Tumour biomechanical response to the vascular disrupting agent ZD6126 in vivo assessed by magnetic resonance elastography. Br J Cancer 110: 1727-1732.

Loveless ME, Lawson D, Collins M, Nadella MV, Reimer C, Huszar D, Halliday J, Waterton JC, Gore JC, Yankeelov TE (2012) Comparisons of the efficacy of a Jak1/2 inhibitor (AZD1480) with a VEGF signaling inhibitor (cediranib) and sham treatments in mouse tumors using DCE-MRI, DW-MRI, and histology. Neoplasia 14: 54-64.

Lyng H, Haraldseth O, Rofstad EK (2000) Measurement of cell density and necrotic fraction in human melanoma xenografts by diffusion weighted magnetic resonance imaging. Magn Reson Med 43: 828-836.

Martin SJ, Reutelingsperger CP, McGahon AJ, Rader JA, van Schie RC, LaFace DM, Green DR (1995) Early redistribution of plasma membrane phosphatidylserine is a general feature of apoptosis regardless of the initiating stimulus: inhibition by overexpression of Bcl-2 and Abl. J Exp Med 182: 1545-1556.

Morse DL, Galons JP, Payne CM, Jennings DL, Day S, Xia G, Gillies RJ (2007) MRI-measured water mobility increases in response to chemotherapy via multiple cell-death mechanisms. NMR Biomed 20: 602-614.

Ng TS, Wert D, Sohi H, Procissi D, Colcher D, Raubitschek AA, Jacobs RE (2013) Serial diffusion MRI to monitor and model treatment response of the targeted nanotherapy CRLX101. Clin Cancer Res 19: 2518-2527.

Nguyen QD, Lavdas I, Gubbins J, Smith G, Fortt R, Carroll LS, Graham MA, Aboagye EO (2013) Temporal and spatial evolution of therapy-induced tumor apoptosis detected by caspase-3-selective molecular imaging. Clin Cancer Res 19: 3914-3924.

Nguyen QD, Smith G, Glaser M, Perumal M, Arstad E, Aboagye EO (2009) Positron emission tomography imaging of drug-induced tumor apoptosis with a caspase-3/7 specific [18F]-labeled isatin sulfonamide. Proc Natl Acad Sci USA 106: 16375-16380.

Patterson DM, Padhani AR, Collins DJ (2008) Technology insight: water diffusion MRI-a potential new biomarker of response to cancer therapy. Nat Clin Pract Oncol 5: 220-233.

Rozel S, Galbán CJ, Nicolay K, Lee KC, Sud S, Neeley C, Snyder LA, Chenevert TL, Rehemtulla A, Ross BD, Pienta KJ (2009) Synergy between anti-CCL2 and docetaxel as determined by DW-MRI in a metastatic bone cancer model. J Cell Biochem 107: 58-64.

Schneider CA, Rasband WS, Eliceiri KW (2012) NIH Image to ImageJ: 25 years of image analysis. Nat Methods 9: 671-675.

Senzer NN, Lorusso P, Martin LP, Schilder RJ, Amaravadi RK, Papadopoulos KP, Segota ZE, Weng DE, Graham M, Adjei AA (2014) Phase II clinical activity and tolerability of the SMAC-mimetic birinapant (TL32711) plus irinotecan in irinotecan-relapsed/refractory metastatic colorectal cancer. 2013 ASCO Annual Meeting.

Sinkus R, Van Beers BE, Vilgrain V, DeSouza N, Waterton JC (2012) Apparent diffusion coefficient from magnetic resonance imaging as a biomarker in oncology drug development. Eur J Cancer 48: 425-431.

Sun YS, Cui Y, Tang L, Qi LP, Wang N, Zhang XY, Cao K, Zhang XP (2011) Early evaluation of cancer response by a new functional biomarker: apparent diffusion coefficient. AJR Am J Roentgenol 197: W23-W29.

Tang L, Zhang XP, Sun YS, Shen L, Li J, Qi LP, Cui Y (2011) Gastrointestinal stromal tumors treated with imatinib mesylate: apparent diffusion coefficient in the evaluation of therapy response in patients. Radiology 258: 729-738.

Thoeny HC, De Keyzer F, Chen F, Vandecaveye V, Verbeken EK, Ahmed B, Sun X, Ni Y, Bosmans H, Hermans R, van Oosterom A, Marchal G, Landuyt W (2005) Diffusion-weighted magnetic resonance imaging allows noninvasive in vivo monitoring of the effects of combretastatin a-4 phosphate after repeated administration. Neoplasia 7: 779-787.

Valonen PK, Lehtimäki KK, Väisänen TH, Kettunen MI, Gröhn OH, Ylä-Herttuala S, Kauppinen RA (2004) Water diffusion in a rat glioma during ganciclovir-thymidine kinase gene therapy-induced programmed 
cell death in vivo: correlation with cell density. J Magn Reson Imaging 19: 389-396.

Vlad RM, Kolios MC, Moseley JL, Czarnota GJ, Brock KK (2010) Evaluating the extent of cell death in 3D high frequency ultrasound by registration with whole-mount tumor histopathology. Med Phys 37: 4288-4297.

Walker-Samuel S, Orton M, McPhail LD, Robinson SP (2009) Robust estimation of the apparent diffusion coefficient (ADC) in heterogeneous solid tumors. Magn Reson Med 62: 420-429.

Waterton JC, Pylkkanen L (2012) Qualification of imaging biomarkers for oncology drug development. Eur J Cancer 48: 409-415.

Workman P, Aboagye EO, Balkwill F, Balmain A, Bruder G, Chaplin DJ, Double JA, Everitt J, Farningham DA, Glennie MJ, Kelland LR, Robinson V, Stratford IJ, Tozer GM, Watson S, Wedge SR, Eccles SA (2010) Guidelines for the welfare and use of animals in cancer research. $\mathrm{Br}$ J Cancer 102(11): 1555-1577.
Zabludoff SD, Deng C, Grondine MR, Sheehy AM, Ashwell S, Caleb BL, Green S, Haye HR, Horn CL, Janetka JW, Liu D, Mouchet E, Ready S, Rosenthal JL, Queva C, Schwartz GK, Taylor KJ, Tse AN, Walker GE, White AM (2008) AZD7762, a novel checkpoint kinase inhibitor, drives checkpoint abrogation and potentiates DNA-targeted therapies. Mol Cancer Ther 7: 2955-2966.

Zietkowski D, Payne GS, Nagy E, Mobberley MA, Ryder TA, deSouza NM (2012) Comparison of NMR lipid profiles in mitotic arrest and apoptosis as indicators of paclitaxel resistance in cervical cell lines. Magn Reson Med 68: $369-377$.

This work is published under the standard license to publish agreement. After 12 months the work will become freely available and the license terms will switch to a Creative Commons AttributionNonCommercial-Share Alike 4.0 Unported License. 